\title{
Precise BER Analysis of Repetition Coded OFDM Systems over Time- and Frequency-Selective Rayleigh Fading Channels
}

\author{
Fumihito SASAMORI ${ }^{\dagger a)}$, Senior Member, Satoru ASADA ${ }^{\dagger \dagger}$, Nonmember, Osamu TAKYU $^{\dagger}$, \\ and Shiro HANDA ${ }^{\dagger}$, Members
}

\begin{abstract}
SUMMARY Orthogonal frequency division multiplexing (OFDM) has great advantages of high spectrum efficiency and robustness against multipath fading. When the received signal is deeply suppressed by deep fading, path loss and shadowing, the received carrier power must be increased in order to avoid degrading communication quality and provide high reliability at the cost of lower system throughput. A repetition coding is very attractive in providing the high reliability with simple configuration and the low decoding complexity of maximal ratio combining. In order to analytically confirm the effectiveness of repetition coded OFDM systems, we theoretically analyze the effect of increasing the number of repetitions (diversity branches) and acquiring both time and frequency diversity gain, and then derive a closed-form equation of average bit error rate (BER) to easily but precisely evaluate the performance.

key words: OFDM, repetition coding, maximal ratio combining, fading correlation, closed-form equation
\end{abstract}

\section{Introduction}

Orthogonal frequency division multiplexing (OFDM) [1], [2], which is applied to various wireless communication systems such as wireless LAN, digital terrestrial TV broadcasting, terrestrial mobile communication and wireless smart sensors networks, has great advantages of high spectrum efficiency and robustness against multipath fading. Many elemental wireless technologies can be combined with OFDM, for example, space-time (or space-frequency) coding for diversity [3]-[5] and error correcting coding (ECC) [6]-[8] to improve communication quality, adaptive modulation [9], [10] and multi-input multi-output (MIMO) [11], [12] to enhance system throughput.

When the received signal is deeply suppressed by deep fading, path loss and shadowing, or when the propagation distance is long, the received carrier power must be increased in order to avoid degrading the communication quality and provide high reliability at the cost of lower system throughput. In this case, ECC is very attractive. Turbo coding and LDPC coding can drastically improve the communication quality with low received power at the cost of decoding complexity. Convolutional coding with Viterbi decoding, which is widely applied in wireless communication systems, is inferior to the turbo coding and the LDPC coding

Manuscript received April 28, 2014.

Manuscript revised July 30, 2014.

${ }^{\dagger}$ The authors are with the Faculty of Engineering, Shinshu University, Nagano-shi, 380-8553 Japan.

${ }^{\dagger \dagger}$ The author is with Central Japan Railway Company, Nagoyashi, 450-6101 Japan.

a)E-mail: fsasa@shinshu-u.ac.jp

DOI: 10.1587/transcom.E98.B.88 in quality, but its decoding complexity is low.

On the other hand, we have paid attention to a repetition coding to provide the high reliability with simple configuration and the low decoding complexity [13]-[15], which is also applied to IEEE 802.16 standards [16]. The repetition coding has the advantage that only one pair of transmit/receive antennas is required and that the decoding process, that is, maximal ratio combining (MRC) is very simple as compared with any ECC. But the repetition coding has the disadvantage that the maximum effect to improve the communication quality is limited. In recent years, Machine-to-Machine (M2M) communications and Internet of Things (IoT) command considerable attention. In wireless smart sensors networks, for example, digital signal processing tasks to improve the communication quality should be implemented on a radio device with limited hardware resources and limited package size. Even if the transmission path is degraded, repetition coding can easily provide high reliability by increasing the number of repetitions.

In this paper, in order to analytically confirm the effectiveness of repetition coded OFDM systems, we theoretically analyze the effect of increasing the number of repetitions (diversity branches) and derive a closed-form equation of average bit error rate (BER). In the case of twobranch MRC in either time domain or frequency domain, we have proposed closed-form equations for calculating the BER in the OFDM systems with a simple repetition coding (RC-OFDM) [13], [14] and an Hermite-symmetric repetition coding (HC-OFDM) [15] considering differential detection [13] and coherent detection [14], [15]. The repetition coding is simple, but needs higher-level modulation to compensate the degradation of the bit rate. The Hermitesymmetric repetition coding realizes the simultaneous transmission of two data sequences with the real part (I-ch) and the imaginary part (Q-ch) of wireless channels individually. Therefore, the higher-level modulation is not necessary, but a phase compensator to correct the IQ imbalance caused by fading is necessary [17], [18]. The number of repetitions is restricted to two and its direction is also restricted to either time or frequency axis in the previous researches [14], [15]. In this paper, we theoretically analyze the effect of not only increasing the number of repetitions but also acquiring both time and frequency diversity gains in order to combat the deteriorated transmission in both RC-OFDM and HC-OFDM systems. By using the derived closed-form equations, the BER improvement can be easily but precisely evaluated. 


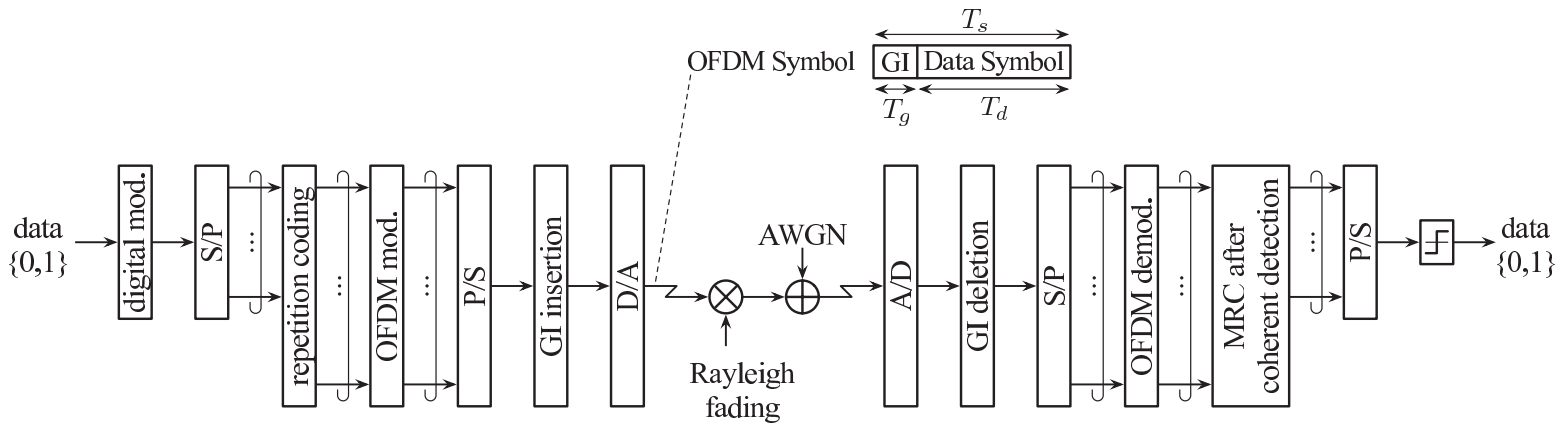

Fig. 1 Block diagram in equivalent low-pass systems.

The remainder of this paper is organized as follows. A system model of the repetition coded OFDM systems and the modified repetition coding methods are described in Sect. 2. Closed-form equations for the BER are derived for the purpose of theoretically verifying the effectiveness of repetition coding over time- and frequency-selective Rayleigh fading channels in Sect. 3. The BER performances are evaluated in Sect.4, and the paper is concluded in Sect. 5.

\section{Repetition Coded OFDM Systems}

In this section, we will explain a system model of repetition coded OFDM systems and modified coding methods to provide high reliability with simple configuration at the cost of lower bit rate even if the transmission path is degraded.

\subsection{System Model}

Figure 1 shows a block diagram of repetition coded OFDM systems, where $T_{s}$ is an OFDM symbol duration including a guard interval $T_{q}$ and a data symbol duration $T_{d}$. At a transmitter, a binary data sequence is converted to digitalmodulated symbols by phase shift keying (PSK) or quadrature amplitude modulation (QAM), which are parallelized by serial-to-parallel (S/P) conversion. We assume a twodimensional array of $N_{t}$ OFDM symbols in a time domain and $N_{f}$ subcarriers in a frequency domain. One digitalmodulated symbol is copied and stored in $L$ arrays according to a simple repetition coding or an Hermite-symmetric repetition coding, which will be mentioned in Sect. 2.2. The number of repetitions $L$ is the same meaning as the number of diversity branches. After OFDM modulation, parallelto-serial $(\mathrm{P} / \mathrm{S})$ conversion, guard interval (GI) insertion and digital-to-analog (D/A) conversion, generated OFDM symbols are transmitted.

The OFDM symbols are subjected to both time- and frequency-selective Rayleigh fading [19] and are added white Gaussian noise (AWGN). It is assumed that intercarrier interference (ICI) caused by Doppler effect and intersymbol interference (ISI) caused by multipath delay spread can be completely compensated just like in [14], [15]. Average carrier-to-noise power ratio (CNR) of the received signal is defined as $\Gamma$. At a receiver, after analog-to-digital
(A/D) conversion, GI deletion, serial-to-parallel (S/P) conversion, OFDM demodulation and coherent detection, $L$ output signals are summed up based on the maximal ratio combining (MRC) with time and/or frequency correlation by fading. A binary data sequence is demodulated after $\mathrm{P} / \mathrm{S}$ conversion and data decision.

In the system evaluation, we assume the same conditions of the received carrier power (the average $\mathrm{CNR} \Gamma$ ), the frequency bandwidth (the number of subcarriers $N_{f}$ ) and the bit rate regardless of the type of repetition coding. Moreover, the perfect channel estimation is assumed in order to theoretically evaluate the optimal effect of the repetition coding upon the performance.

\subsection{Repetition Coding}

In the case of two-branch MRC $(L=2)$, we have proposed closed-form equations for calculating average bit error rate (BER) of the OFDM systems with a simple repetition coding [14] and an Hermite-symmetric repetition coding [15] in either time domain or frequency domain. When the received signal is deeply suppressed by deep fading, path loss and shadowing, or when the propagation distance is long, the received carrier power must be increased in order to avoid degrading the BER and provide high reliability at the cost of lower bit rate. In order to 'easily' solve the problem with the simple configuration, we enhance the repetition coding by

- increasing the number of repetitions (branches) $L$,

- acquiring both time and frequency diversity gains, namely, two-dimensional diversity gain.

In this paper, we aim to derive closed-form equations for calculating the average BER by the above solution and confirm the effectiveness of MRC. It should be noted that the upper limit of $L$ is four because of the mathematical restriction to analytically solve an equation, which will be mentioned in Sect. 3.

Now we concretely explain the modified repetition coding methods.

\subsubsection{Simple Repetition Coding}

Figure 2 shows a concept of the simple repetition coding in 


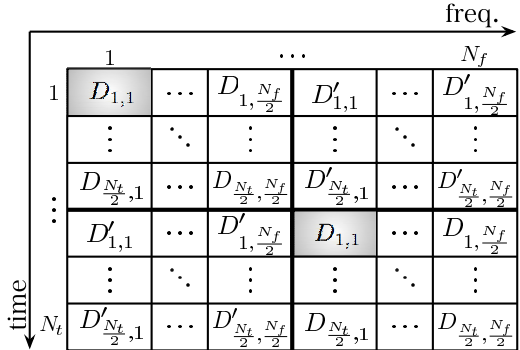

(a) $L=2$

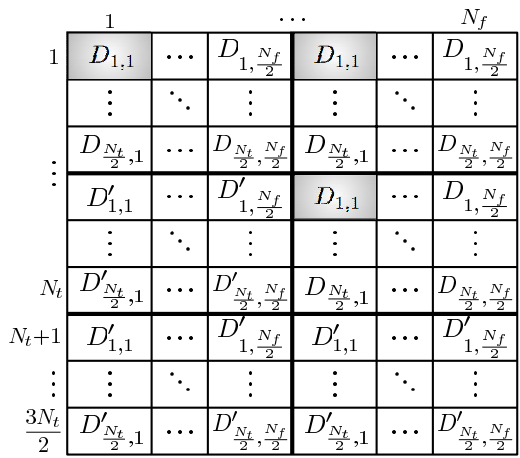

(b) $L=3$
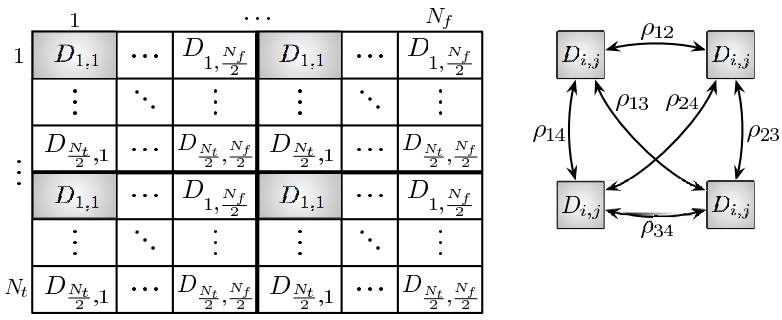

(c) $L=4$

Fig. 2 Simple repetition coding to acquire two-dimensional diversity gain and its fading correlation.

order to acquire the two-dimensional diversity gain and its fading correlation, where $D_{i, j}$ and $D_{i, j}^{\prime}$ are digital-modulated symbols by PSK or QAM. The fading correlation $\rho_{l k}$ is a key parameter to determine the BER. The two-dimensional array of $N_{t}$ OFDM symbols and $N_{f}$ subcarriers is divided into four regions. The symbols of the same label are to be combined at the receiver. For example, a set of the symbols $D_{1,1}$ is focused in Fig. 2. The cycle period of the repetition coding becomes $N_{t}$ OFDM symbols in the case of $L=2,4$ and $3 N_{t} / 2$ OFDM symbols in the case of $L=3$.

In this paper, we would like to also examine the effect of increasing $L$ with either time or frequency diversity gain (one-dimensional diversity gain) as shown in Fig. 3, where $D_{i}$ is a digital-modulated symbol. The variable $N$ is the same as $N_{t}$ when considering the time diversity gain and $N_{f}$ when considering the frequency diversity gain. It is noted that the coding in Fig. 3(a) is the same as in [14] and the others in Figs. 2 and 3 are the enhanced methods in this paper.

Average CNR $\Gamma_{s}$ of each digital-modulated symbol in the simple repetition coded OFDM (RC-OFDM) systems can be expressed as

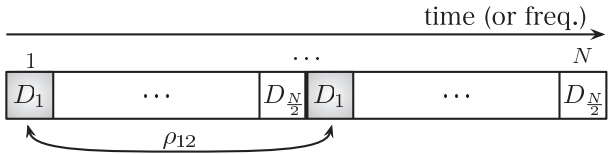

(a) $L=2$

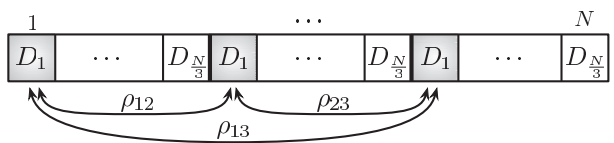

(b) $L=3$

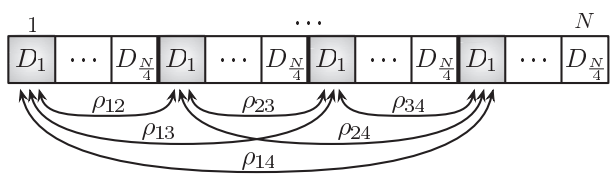

(c) $L=4$

Fig. 3 Simple repetition coding to acquire one-dimensional diversity gain and its fading correlation.

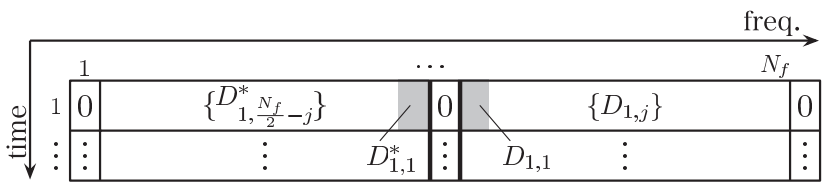

(a) frequency diversity $\left(L=2\right.$ and $\left.j=1,2, \cdots, \frac{N_{f}}{2}-1\right)$

\begin{tabular}{|c|c|c|c|c|}
\hline 10 & $\left\{D_{1, \frac{N_{f}}{2}-j}^{*}\right\}$ & 0 & $\left\{D_{1, j}\right\}$ & 0 \\
\hline$\vdots$ & & : & $D_{1,1}$ & $\vdots$ \\
\hline 0 & $\left\{D_{\frac{N_{t}}{2}, \frac{N_{f}}{2}-j}^{*}\right\}$ & 0 & $\left\{D_{\frac{N_{t}}{2}, j}\right\}$ & 0 \\
\hline 0 & $\left\{D_{1, \frac{N_{f}}{2}-j}^{*}\right\}$ & 0 & $\left\{D_{1, j}\right\}$ & 0 \\
\hline$\vdots$ & $\vdots$ & $\vdots$ & $\vdots$ & $\vdots$ \\
\hline 0 & $\left\{D_{\frac{N_{t}}{2}, \frac{N_{f}}{2}-j}^{*}\right\}$ & 0 & $\left\{D_{\frac{N_{t}}{2}, j}\right\}$ & 0 \\
\hline
\end{tabular}

(b) two-dimensional diversity ( $L=4$ and $\left.j=1,2, \cdots, \frac{N_{f}}{2}-1\right)$

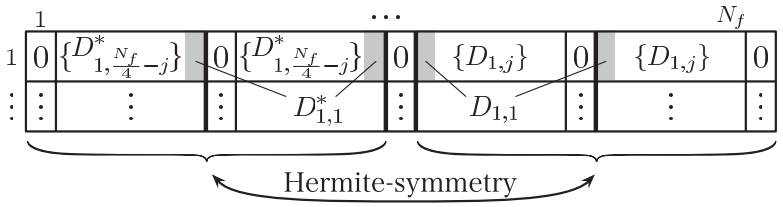

(c) frequency diversity $\left(L=4\right.$ and $\left.j=1,2, \cdots, \frac{N_{f}}{4}-1\right)$

Fig. 4 Hermite-symmetric repetition coding.

$$
\Gamma_{s}=\frac{\Gamma}{1+\alpha},
$$

where $\alpha\left(=T_{g} / T_{d}\right)$ is the GI ratio.

\subsubsection{Hermite-Symmetric Repetition Coding}

Figure 4 shows a concept of the Hermite-symmetric repetition coding, where the sequence $\left\{D_{i, j}^{*}\right\}$ is the complex conjugate sequence of $\left\{D_{i, j}\right\}$ with Hermite symmetry which includes the $\left(N_{f}+1\right)$-th guard subcarrier. For example, a set of the symbols $D_{1,1}$ and $D_{1,1}^{*}$ is focused in the figure. It is noted that the coding in Fig. 4(a) is the same as in [15] and the other two are the enhanced methods in this paper, and 
that three-branch MRC $(L=3)$ cannot be constructed considering the symmetry.

Average CNR $\Gamma_{s}$ of each digital-modulated symbol in the Hermite-symmetric repetition coded OFDM (HCOFDM) can be expressed as [15]

$$
\Gamma_{s}=\frac{\Gamma}{2(1+\alpha)} .
$$

The $\Gamma_{s}$ in the HC-OFDM systems, which realize the simultaneous transmission of two data sequences with both IQ wireless channels individually, should be reduced by half as compared with that in the RC-OFDM systems in order to keep the same condition of the received carrier power.

\section{Average BER Analysis}

In this section, we will derive closed-form equations for calculating the average BER in the RC-OFDM systems and the HC-OFDM systems explained in Sect. 2. When the average BER is analyzed considering the MRC with fading correlation, the correlated signals with the equal average $\mathrm{CNR} \Gamma_{s}$ at all $L$ branches should be decoupled into the uncorrelated signals with the individual average $\mathrm{CNRs}_{l}(l=1,2, \cdots, L)$ by eigenvector transformation [20]. First of all, we will explain the fading correlation.

\subsection{Fading Correlation Function}

We would like to briefly quote the functions for calculating the fading correlation.

A time correlation between signals with a time interval of $\Delta_{T}$ OFDM symbols can be calculated by [19]

$$
\rho_{t}\left(\Delta_{T}\right)=\mathrm{J}_{0}\left(2 \pi \Delta_{T} f_{D} T_{s}\right),
$$

where $f_{D} T_{s}$ is the maximum Doppler frequency normalized by OFDM symbol frequency, and $\mathrm{J}_{0}(\cdot)$ is the Bessel function of zeroth order. A frequency correlation between signals with a frequency interval of $\Delta_{F}$ subcarriers can be calculated by [14]

$$
\rho_{f}\left(\Delta_{F}\right)=\sum_{n=0}^{N_{g}-1} \frac{p_{r}[n]}{\sum_{m=0} p_{r}[m]} \exp \left\{-j 2 \pi \Delta_{F}(1+\alpha) \frac{\tau[n]}{T_{s}}\right\},
$$

where $p_{r}[n]$ is a received power with a time delay $\tau[n]$ in a discrete-time power delay profile, $N_{g}$ is a sample size in the GI. Figure 5 shows a concept of the discrete-time power delay profile. The GI is assumed to be longer than the maximum multipath delay $\tau\left[N_{q}-1\right]$ in order to avoid the ISI, which is mentioned in Sect. 2.1. By using (4), the frequency correlation can be precisely calculated from any discretetime power delay profile. On the other hand, we can utilize a simple function [20]

$$
\rho_{f}\left(\Delta_{F}\right)=\frac{1-j 2 \pi \Delta_{F}(1+\alpha) \frac{\sigma_{\tau}}{T_{s}}}{1+\left\{2 \pi \Delta_{F}(1+\alpha) \frac{\sigma_{\tau}}{T_{s}}\right\}^{2}},
$$

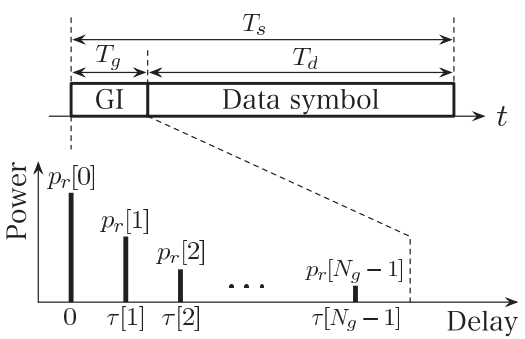

Fig. 5 Discrete-time power delay profile.

where $\sigma_{\tau}$ is rms delay spread. It should be noted that the simple function (5) includes calculation error when using the large frequency interval $\Delta_{F}$, and that the error becomes smaller in the case of the larger FFT point size [14].

In order to analyze the effect of the two-dimensional diversity gain, the time-frequency correlation should be discussed here. The time correlation $\rho_{t}\left(\Delta_{T}\right)$ caused by Doppler effect and the frequency correlation $\rho_{f}\left(\Delta_{F}\right)$ caused by multipath delay spread are mutually independent because the two are mutually independent events. Therefore, the timefrequency correlation between signals with the time interval of $\Delta_{T}$ and the frequency interval of $\Delta_{F}$ can be expressed as $\rho_{t}\left(\Delta_{T}\right) \cdot \rho_{f}\left(\Delta_{F}\right)$.

\subsubsection{Simple Repetition Coding}

By using the functions (3), (4) and (5), we can summarize the fading correlation $\rho_{l k}$ between repetition coded symbols in Figs. 2 and 3 as follows:

1. $L=2$

- two-dimensional diversity correlation:

$$
\rho_{12}=\rho_{t}\left(\frac{N_{t}}{2}\right) \cdot \rho_{f}\left(\frac{N_{f}}{2}\right) \text {. }
$$

- one-dimensional time diversity correlation:

$$
\rho_{12}=\rho_{t}\left(\frac{N_{t}}{2}\right) .
$$

- one-dimensional frequency diversity correlation:

$$
\rho_{12}=\rho_{f}\left(\frac{N_{f}}{2}\right) \text {. }
$$

Equations (7) and (8) are the same expressions in [14].

2. $L=3$

- two-dimensional diversity correlation:

$$
\begin{aligned}
& \rho_{12}=\rho_{f}\left(\frac{N_{f}}{2}\right), \\
& \rho_{13}=\rho_{t}\left(\frac{N_{t}}{2}\right) \cdot \rho_{f}\left(\frac{N_{f}}{2}\right), \\
& \rho_{23}=\rho_{t}\left(\frac{N_{t}}{2}\right) .
\end{aligned}
$$


- one-dimensional time diversity correlation:

$$
\begin{aligned}
& \rho_{12}=\rho_{23}=\rho_{t}\left(\frac{N_{t}}{3}\right), \\
& \rho_{13}=\rho_{t}\left(\frac{2 N_{t}}{3}\right) .
\end{aligned}
$$

- one-dimensional frequency diversity correlation:

$$
\begin{aligned}
& \rho_{12}=\rho_{23}=\rho_{f}\left(\frac{N_{f}}{3}\right), \\
& \rho_{13}=\rho_{f}\left(\frac{2 N_{f}}{3}\right) .
\end{aligned}
$$

3. $L=4$

- two-dimensional diversity correlation:

$$
\begin{aligned}
& \rho_{12}=\rho_{34}=\rho_{f}\left(\frac{N_{f}}{2}\right), \\
& \rho_{13}=\rho_{24}=\rho_{t}\left(\frac{N_{t}}{2}\right) \cdot \rho_{f}\left(\frac{N_{f}}{2}\right), \\
& \rho_{14}=\rho_{23}=\rho_{t}\left(\frac{N_{t}}{2}\right) .
\end{aligned}
$$

- one-dimensional time diversity correlation:

$$
\begin{aligned}
& \rho_{12}=\rho_{23}=\rho_{34}=\rho_{t}\left(\frac{N_{t}}{4}\right), \\
& \rho_{13}=\rho_{24}=\rho_{t}\left(\frac{N_{t}}{2}\right), \\
& \rho_{14}=\rho_{t}\left(\frac{3 N_{t}}{4}\right) .
\end{aligned}
$$

- one-dimensional frequency diversity correlation:

$$
\begin{aligned}
& \rho_{12}=\rho_{23}=\rho_{34}=\rho_{f}\left(\frac{N_{f}}{4}\right), \\
& \rho_{13}=\rho_{24}=\rho_{f}\left(\frac{N_{f}}{2}\right), \\
& \rho_{14}=\rho_{f}\left(\frac{3 N_{f}}{4}\right) .
\end{aligned}
$$

It is noted in the simple repetition coding that every set of symbols $D_{i, j}$ has the same fading correlation.

\subsubsection{Hermite-Symmetric Repetition Coding}

A definition of the fading correlation $\rho_{l k}$ between symbols to be combined is the same as that in the simple repetition coding. That is, the correlations in Figs. 4(a) and (c) correspond to those in Figs. 3(a) and (c) respectively, and the correlation in Fig. 4(b) corresponds to that in Fig. 2(c). Unlike in the simple repetition coding, the frequency interval between symbols varies depending on the variable $j$ in the Hermite-symmetric repetition coding. We can summarize the fading correlation as follows:

1. frequency diversity correlation in $L=2$ [15]

$$
\rho_{12}=\rho_{f}(2 j), \quad j=1,2, \cdots, \frac{N_{f}}{2}-1 .
$$

2. two-dimensional diversity correlation in $L=4$

$$
\begin{aligned}
& \rho_{12}=\rho_{34}=\rho_{f}(2 j), \\
& \rho_{13}=\rho_{24}=\rho_{t}\left(\frac{N_{t}}{2}\right) \cdot \rho_{f}(2 j), \\
& \rho_{14}=\rho_{23}=\rho_{t}\left(\frac{N_{t}}{2}\right), \quad j=1,2, \cdots, \frac{N_{f}}{2}-1 .
\end{aligned}
$$

3. frequency diversity correlation in $L=4$

$$
\begin{aligned}
& \rho_{12}=\rho_{34}=\rho_{f}\left(\frac{N_{f}}{4}\right), \\
& \rho_{23}=\rho_{f}(2 j), \\
& \rho_{13}=\rho_{24}=\rho_{f}\left(\frac{N_{f}}{4}+2 j\right), \\
& \rho_{14}=\rho_{f}\left(\frac{N_{f}}{2}+2 j\right), \quad j=1,2, \cdots, \frac{N_{f}}{4}-1 .
\end{aligned}
$$

\subsection{Eigenvector Transformation}

Now we explain how to calculate the individual average CNRs $\Gamma_{l}(l=1,2, \cdots, L)$ of the uncorrelated signals decoupled by eigenvector transformation. We would like to solve an eigenvalue equation by an analytical method, but there is no formula for general quintic equations. Therefore, the upper limit of $L$ is forced to be four. The analytical solution is introduced in this section.

\subsection{1 $L=2$}

A correlation matrix $C$ is expressed as

$$
C=\left[\begin{array}{cc}
1 & \rho_{12} \\
\rho_{12}^{*} & 1
\end{array}\right]
$$

where $\rho_{l k}^{*}$ is a complex conjugation of $\rho_{l k}$. The eigenvalues $\lambda_{1}$ and $\lambda_{2}$ can be obtained as

$$
\begin{aligned}
& \lambda_{1}=1+\left|\rho_{12}\right|, \\
& \lambda_{2}=1-\left|\rho_{12}\right| .
\end{aligned}
$$

Therefore, the individual average $\mathrm{CNRs} \Gamma_{i}$ become

$$
\Gamma_{i}=\lambda_{i} \Gamma_{s}, \quad i=1,2 .
$$

Equation (20) is the same expression in [14], [15] regardless of the number of diversity dimension.

\subsection{2 $L=3$}

A correlation matrix $C$ is expressed as

$$
C=\left[\begin{array}{ccc}
1 & \rho_{12} & \rho_{13} \\
\rho_{12}^{*} & 1 & \rho_{23} \\
\rho_{13}^{*} & \rho_{23}^{*} & 1
\end{array}\right] .
$$


The eigenvalues $\lambda_{l}(l=1,2,3)$ can be obtained by solving the following cubic equation:

$$
\begin{aligned}
& (1-\lambda)^{3}-\left(\left|\rho_{12}\right|^{2}+\left|\rho_{13}\right|^{2}+\left|\rho_{23}\right|^{2}\right)(1-\lambda) \\
& \quad+2 \operatorname{Re}\left(\rho_{12} \rho_{13}^{*} \rho_{23}\right)=0 \\
& \therefore \lambda^{3}-3 \lambda^{2}+\left(3-\left|\rho_{12}\right|^{2}-\left|\rho_{13}\right|^{2}-\left|\rho_{23}\right|^{2}\right) \lambda \\
& \quad+\left|\rho_{12}\right|^{2}+\left|\rho_{13}\right|^{2}+\left|\rho_{23}\right|^{2}-2 \operatorname{Re}\left(\rho_{12} \rho_{13}^{*} \rho_{23}\right)-1=0,
\end{aligned}
$$

where $\operatorname{Re}(x)$ returns the real part of a complex number $x$. By setting

$$
\begin{aligned}
& a_{2}=-3 \\
& a_{1}=3-\left|\rho_{12}\right|^{2}-\left|\rho_{13}\right|^{2}-\left|\rho_{23}\right|^{2} \\
& a_{0}=\left|\rho_{12}\right|^{2}+\left|\rho_{13}\right|^{2}+\left|\rho_{23}\right|^{2}-2 \operatorname{Re}\left(\rho_{12} \rho_{13}^{*} \rho_{23}\right)-1,
\end{aligned}
$$

the eigenvalues $\lambda_{i}(i=1,2,3)$ can be obtained by using Cardano's solution for the cubic equation in Appendix A. Finally, the individual average $\mathrm{CNRs} \Gamma_{i}$ become

$$
\Gamma_{i}=\lambda_{i} \Gamma_{s}, \quad i=1,2,3 .
$$

\subsection{3 $L=4$}

A correlation matrix $C$ is expressed as

$$
C=\left[\begin{array}{cccc}
1 & \rho_{12} & \rho_{13} & \rho_{14} \\
\rho_{12}^{*} & 1 & \rho_{23} & \rho_{24} \\
\rho_{13}^{*} & \rho_{23}^{*} & 1 & \rho_{34} \\
\rho_{14}^{*} & \rho_{24}^{*} & \rho_{34}^{*} & 1
\end{array}\right] .
$$

The eigenvalues $\lambda_{l}(l=1,2,3,4)$ can be obtained by solving the following quartic equation:

$$
\begin{aligned}
(1-\lambda)^{4} & \\
- & \left(\left|\rho_{12}\right|^{2}+\left|\rho_{13}\right|^{2}+\left|\rho_{14}\right|^{2}\right. \\
& \left.+\left|\rho_{23}\right|^{2}+\left|\rho_{24}\right|^{2}+\left|\rho_{34}\right|^{2}\right)(1-\lambda)^{2} \\
+ & 2\left\{\operatorname{Re}\left(\rho_{12} \rho_{13}^{*} \rho_{23}\right)+\operatorname{Re}\left(\rho_{12} \rho_{14}^{*} \rho_{24}\right)\right. \\
& \left.+\operatorname{Re}\left(\rho_{13} \rho_{14}^{*} \rho_{34}\right)+\operatorname{Re}\left(\rho_{23} \rho_{24}^{*} \rho_{34}\right)\right\}(1-\lambda) \\
+ & \left|\rho_{12}\right|^{2}\left|\rho_{34}\right|^{2}+\left|\rho_{13}\right|^{2}\left|\rho_{24}\right|^{2}+\left|\rho_{14}\right|^{2}\left|\rho_{23}\right|^{2} \\
- & 2\left\{\operatorname{Re}\left(\rho_{12} \rho_{13}^{*} \rho_{24} \rho_{34}^{*}\right)+\operatorname{Re}\left(\rho_{12} \rho_{14}^{*} \rho_{23} \rho_{34}\right)\right. \\
& \left.+\operatorname{Re}\left(\rho_{13} \rho_{14}^{*} \rho_{23}^{*} \rho_{24}\right)\right\}=0
\end{aligned}
$$

By setting

$$
\begin{aligned}
y=1 & -\lambda \\
b_{2}= & -\left(\left|\rho_{12}\right|^{2}+\left|\rho_{13}\right|^{2}+\left|\rho_{14}\right|^{2}+\left|\rho_{23}\right|^{2}+\left|\rho_{24}\right|^{2}+\left|\rho_{34}\right|^{2}\right) \\
b_{1}= & 2\left\{\operatorname{Re}\left(\rho_{12} \rho_{13}^{*} \rho_{23}\right)+\operatorname{Re}\left(\rho_{12} \rho_{14}^{*} \rho_{24}\right)\right. \\
& \left.+\operatorname{Re}\left(\rho_{13} \rho_{14}^{*} \rho_{34}\right)+\operatorname{Re}\left(\rho_{23} \rho_{24}^{*} \rho_{34}\right)\right\} \\
b_{0}= & \left|\rho_{12}\right|^{2}\left|\rho_{34}\right|^{2}+\left|\rho_{13}\right|^{2}\left|\rho_{24}\right|^{2}+\left|\rho_{14}\right|^{2}\left|\rho_{23}\right|^{2} \\
& -2\left\{\operatorname{Re}\left(\rho_{12} \rho_{13}^{*} \rho_{24} \rho_{34}^{*}\right)+\operatorname{Re}\left(\rho_{12} \rho_{14}^{*} \rho_{23} \rho_{34}\right)\right. \\
& \left.+\operatorname{Re}\left(\rho_{13} \rho_{14}^{*} \rho_{23}^{*} \rho_{24}\right)\right\},
\end{aligned}
$$

the quartic Eq. (26) can be rewritten as

$$
y^{4}+b_{2} y^{2}+b_{1} y+b_{0}=0 .
$$

The solutions $y_{i}(i=1,2,3,4)$ can be obtained by using Euler's solution for the quartic equation in Appendix B. Finally, the individual average $\mathrm{CNRs} \Gamma_{i}$ become

$$
\Gamma_{i}=\lambda_{i} \Gamma_{s}=\left(1-y_{i}\right) \Gamma_{s}, \quad i=1,2,3,4 .
$$

\subsection{Average BER in RC-OFDM Systems}

An average BER $\bar{P}_{b}$ with Gray-mapped $M$-PSK or $M$-QAM over fading channels can be calculated by [19], [21]

$$
\bar{P}_{b}=\int_{0}^{\infty} p(\gamma) P_{b}(\gamma) d \gamma,
$$

where $\gamma$ is CNR of a received signal, $p(\gamma)$ is a probability density function (PDF) of $\gamma, P_{b}(\gamma)$ is an expression of BER over AWGN channels as follows:

$$
P_{b}(\gamma)=A \operatorname{erfc}(\sqrt{B \gamma}) .
$$

Values of $A$ and $B$ in each modulation are appeared in Table 1 , and $\operatorname{erfc}(\cdot)$ is a complementary error function. Moreover, the average CNR over fading channels can be defined as

$$
\Gamma=\int_{0}^{\infty} \gamma p(\gamma) d \gamma .
$$

The PDF of the CNR $\gamma$ given by the correlated $L$ branch MRC can be expressed as [22]

$$
p(\gamma)=\frac{1}{\prod_{l=1}^{L} \Gamma_{l}} \sum_{l=1}^{L} \frac{1}{\prod_{\substack{k=1 \\ k \neq l}}^{L}\left(\frac{1}{\Gamma_{k}}-\frac{1}{\Gamma_{l}}\right)} \exp \left(-\frac{\gamma}{\Gamma_{l}}\right) .
$$

By substituting (33) for (30), the closed-form equation for the average BER in the RC-OFDM systems becomes

$$
\bar{P}_{b}=A \sum_{l=1}^{L} \frac{1}{\prod_{\substack{k=1 \\ k \neq l}}^{L}\left(1-\frac{\Gamma_{k}}{\Gamma_{l}}\right)}\left(1-\frac{1}{\sqrt{1+\frac{1}{B \Gamma_{l}}}}\right) .
$$

It is noted that the following formulas are utilized to derive (34): [23]

$$
\begin{aligned}
& \operatorname{erfc}(x)=1-\operatorname{erf}(x), \\
& \int_{0}^{\infty} \exp (-a x) \operatorname{erf}(\sqrt{b x}) d x=\frac{1}{a} \sqrt{\frac{b}{a+b} .}
\end{aligned}
$$

When the same value appears among $\Gamma_{l}$ in (34), a convenient method to prevent division by zero is explained in [20, p.125]. By substituting $L=2$ for (34), the same equation for the average BER in [14] can be derived. 
Table 1 Values of $A$ and $B$.

\begin{tabular}{c|c|c}
\hline \hline Modulation & $A$ & $B$ \\
\hline \hline BPSK & $1 / 2$ & 1 \\
\hline QPSK & $1 / 2$ & $1 / 2$ \\
\hline 8PSK & $1 / 3$ & $\sin ^{2}(\pi / 8)$ \\
\hline 16QAM & $3 / 8$ & $1 / 10$ \\
\hline 64QAM & $7 / 24$ & $1 / 42$ \\
\hline 256QAM & $15 / 64$ & $1 / 170$ \\
\hline \hline
\end{tabular}

\subsection{Average BER in HC-OFDM Systems}

Since the frequency interval between symbols $D_{i, j}$ varies depending on the variable $j$ in Fig. 4, the fading correlation and the resulting BER also vary by symbol. Therefore, each average BER in (34) should be calculated according to each variable $j$ in (15), (16) and (17). The overall average BER in the HC-OFDM systems can be derived by averaging the BER for every $j$.

\subsection{Average BER without Fading Correlation}

When the digital-modulated symbols with MRC can be considered to be mutually independent, the maximum diversity gain can be achieved. In order to examine a lower bound on the average BER performance, we introduce a closed-form equation for the average BER without fading correlation.

The PDF of the CNR $\gamma$ given by the independent $L$ branch MRC can be expressed as [19]

$$
p(\gamma)=\frac{r^{L-1}}{\Gamma^{L}(L-1) !} \exp \left(-\frac{\gamma}{\Gamma}\right) .
$$

By using (30), the desired BER equation can be derived as follows:

$$
\begin{aligned}
\bar{P}_{b} & =\frac{A}{\Gamma^{L}(L-1) !} \int_{0}^{\infty} \gamma^{L-1} \operatorname{erfc}(\sqrt{B \gamma}) \exp \left(-\frac{\gamma}{\Gamma}\right) d \gamma \\
& =A\left\{1-\frac{1}{\sqrt{1+\frac{1}{B \Gamma}}} \sum_{l=0}^{L-1} \frac{(2 l-1) ! !}{2^{l} \cdot l !} \cdot \frac{1}{(1+B \Gamma)^{l}}\right\} .
\end{aligned}
$$

The derivation method is beyond the scope of this paper, then we omit it for want of space.

\section{BER Evaluation}

First of all, in order to verify the theoretical analysis in Sect. 3, we compare performances obtained by the derived analytical equations and those by Monte Carlo simulation. Table 2 shows the simulation parameters. In the simulation, a tapped-delay line channel simulator is considered as the fading channel [24], and each channel tap is simulated using the Jakes' model [19]. An exponential decay power delay profile is assumed [25] in the multipath fading channel model as shown in Fig. 5. The number of multipath components is the same as $N_{g}$. The digital modulation is assumed
Table 2 Simulation parameters

\begin{tabular}{c|c}
\hline \hline Modulation & $\begin{array}{c}\text { 16QAM for RC-OFDM } \\
\text { QPSK for HC-OFDM }\end{array}$ \\
\hline Detection & Ideal coherent detection \\
\hline FFT point size & 64 \\
\hline Number of subcarriers $N_{f}$ & 48 \\
\hline Number of OFDM symbols $N_{t}$ & 12 \\
\hline Number of GI samples $N_{g}$ & 16 \\
\hline GI ratio $\alpha$ & $1 / 4$ \\
\hline Noise & AWGN \\
\hline Fading & Rayleigh fading \\
\hline Channel model & Exponential decay model \\
\hline \hline
\end{tabular}

Table 3 Example of values of $f_{D} T_{s}$ and $\sigma_{\tau} / T_{s}$ in case of $R=2$.

\begin{tabular}{c|c|c}
\hline \hline & Example 1 & Example 2 \\
\hline Carrier frequency $f_{c}$ & $920 \mathrm{MHz}$ & $2 \mathrm{GHz}$ \\
\hline Bit rate $f_{b}$ & $200 \mathrm{kbps}$ & $10 \mathrm{Mbps}$ \\
\hline $\begin{array}{c}\text { Channel model } \\
\left(\text { rms delay spread } \sigma_{\tau}\right)\end{array}$ & $\begin{array}{c}\text { Channel C } \\
(150 \mathrm{nsec})\end{array}$ & $\begin{array}{c}\text { Channel E-ter } \\
(250 \mathrm{nsec})\end{array}$ \\
\hline Mobile speed $v$ & $10 \mathrm{~km} / \mathrm{h}$ & $60 \mathrm{~km} / \mathrm{h}$ \\
\hline Max. Doppler frequency $f_{D}$ & $8.52 \mathrm{~Hz}$ & $111 \mathrm{~Hz}$ \\
\hline OFDM symbol duration $T_{s}$ & $0.6 \mathrm{msec}$ & $12 \mu \mathrm{sec}$ \\
\hline$f_{D} T_{s}$ & $5.1 \times 10^{-3}$ & $1.3 \times 10^{-3}$ \\
\hline$\sigma_{\tau} / T_{s}$ & $2.5 \times 10^{-4}$ & $2.1 \times 10^{-2}$ \\
\hline \hline
\end{tabular}

to be 16QAM in the RC-OFDM systems. The HC-OFDM systems have the advantage that both IQ wireless channels can be utilized to transmit the two data sequences simultaneously, then the digital modulation in each channel comes to be QPSK considering the same bit rate. On the other hand, it is confirmed by comparing (1) and (2) that the HC-OFDM systems have the disadvantage in the carrier power.

Key parameters on the diversity gain are the maximum Doppler frequency normalized by OFDM symbol frequency $f_{D} T_{s}$ and the rms delay spread normalized by OFDM symbol duration $\sigma_{\tau} / T_{s}$. The $f_{D}$ can be calculated by

$$
f_{D}=\frac{v \times f_{c}}{c},
$$

where $v$ is mobile speed, $c$ is light speed, and $f_{c}$ is carrier frequency. Generally, the OFDM with $N_{f}$ subcarriers can lengthen the symbol duration on each subcarrier by $N_{f}$ times, then the OFDM symbol duration $T_{s}$ including the GI can be calculated by

$$
T_{s}=(1+\alpha) \times N_{f} \times t_{s}=\frac{(1+\alpha) N_{f}}{f_{b} / R},
$$

where $t_{s}$ is the symbol duration of digital-modulated symbol, $f_{b}$ is its bit rate. According to [15], the bit rate per symbol $R$ in $M$-PSK or $M$-QAM considering the number of repetitions $L$ can be easily extended to

$$
R= \begin{cases}\frac{1}{L} \log _{2} M & \text { (for RC-OFDM) } \\ \frac{2}{L}\left(1-\frac{2}{N_{f}}\right) \log _{2} M & \text { (for HC-OFDM) }\end{cases}
$$

As a simple example, Table 3 concretely shows the values 
of $f_{D} T_{s}$ and $\sigma_{\tau} / T_{s}$ when assuming the ETSI/BRAN Channel $\mathrm{C}$ model (mixed outdoor/large indoor area environment at $10 \mathrm{~km} / \mathrm{h}$ ) and Channel E-ter model (rural environment at $60 \mathrm{~km} / \mathrm{h}$ ) [26] in the case of $R=2$. Examples 1 and 2 are based on wireless smart sensors networks and cellular networks, respectively.

\subsection{Verification of Equations}

Figure 6 shows average BER performances in the repetition coded OFDM systems with various coding methods mentioned in Sect. 2.2 under various fading channel conditions. Simulation results are plotted with symbols and analytical results are with lines in the figures. The labels 't-div' and
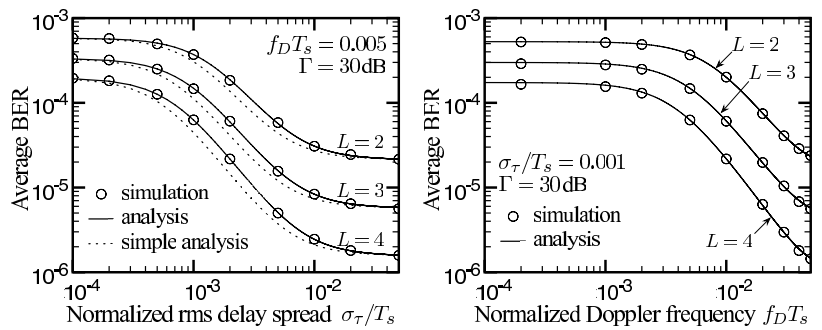

(a) RC-OFDM with two-dimensional diversity
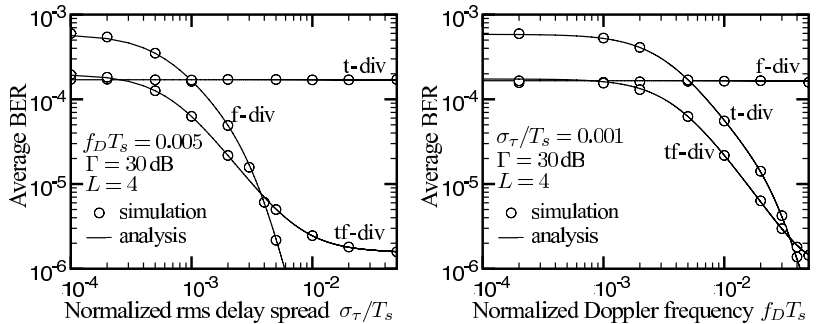

(b) RC-OFDM with $L=4$
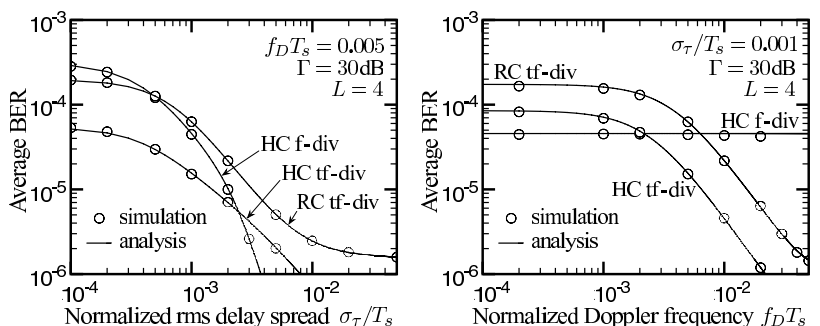

(c) RC-OFDM and HC-OFDM with $L=4$

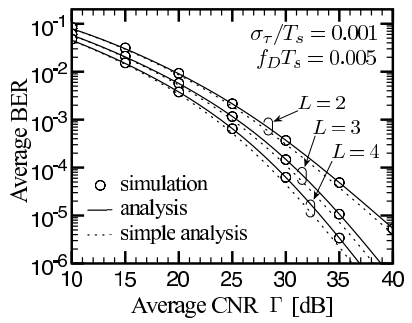

(d) RC-OFDM with two-dimensional diversity

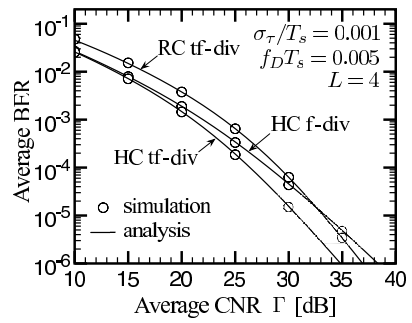

(e) RC-OFDM and HC-OFDM with $L=4$
Fig. 6 Average BER performances of RC-OFDM and HC-OFDM with various coding methods under various fading channel conditions. 'f-div' mean the time diversity and the frequency diversity respectively, which belong to the one-dimensional diversity. The label 'tf-div' means both time and frequency diversities, which belongs to the two-dimensional diversity.

We would like to emphasize that the simulation results precisely coincide with the analytical ones under any condition in Fig. 6, then the average BER analysis in Sect. 3 can be confirmed. It is qualitatively well known that the large diversity gain can be achieved over fading channels with the large delay spread and/or the large Doppler frequency, and with many diversity branches. By using the analytical equation, we can easily but quantitatively verify the superiority or inferiority of each diversity method. It should be noted that the HC-OFDM systems generally outperform the RC-OFDM systems, while the HC-OFDM systems have the computational complexity to compensate the IQ imbalance caused by fading [17], [18].

As for the frequency diversity, we can calculate the correlation by using the simple function (5), whose results are illustrated in Figs. 6(a) and (d). It is found from the figures that the simple function yields slight error, while the simple function has the advantage that we can easily calculate the frequency correlation with only the value of the rms delay spread normalized by OFDM symbol duration $\sigma_{\tau} / T_{s}$.

\subsection{An Example of Usage of Equations}

As an example of the usage of the analytical equations, we compare the effect of repetition coding with that of error correcting coding in order to confirm the effectiveness of MRC. Generally, the error correcting coding can drastically improve BER when the fading correlation among the codeword is low in mobile cellular networks, for example. On the other hand, the fading correlation comes to be large and then the BER improvement becomes small in wireless smart sensors networks, for example. In the latter case, the analytical equations are useful to evaluate the effect of MRC by the repetition coding because of the easy implementation and the low decoding complexity. Figures 7 and 8 compare the average BER performances considering variation of the delay spread and the average CNR, respectively. As the error correcting coding with soft-input (soft-decision) decoding algorithms, we select convolutional coding with Viterbi decoding (constraint length $K=3$ and $K=7$ ) and LDPC coding (rate-1/2 $(3,6)$ regular LDPC codes and the number of iteration is 20). We use an s-random block interleaver to randomize a bit sequence included in a two-dimensional array of $N_{t}$ OFDM symbols and $N_{f}$ subcarriers. It should be noted in $L=4$ that the error correcting coding with code rate $1 / 2$ and the simple repetition coding with $L=2$ are combined. Simulation results by the error correcting coding are plotted with symbols and lines, and analytical results by the repetition coding are with lines in the figures.

In the case of small fading fluctuation $\left(\sigma_{\tau} / T_{s}\right)$ and small $\Gamma$ in $L=2$, the repetition coding outperforms the error correcting coding. But the BER improvement by the repetition coding is saturated in the case of the larger fad- 


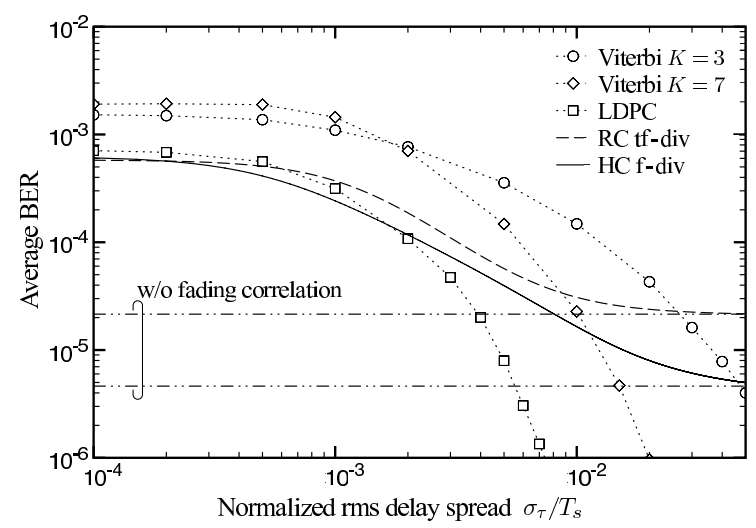

(a) $L=2, \Gamma=30 \mathrm{~dB}, f_{D} T_{s}=0.005$

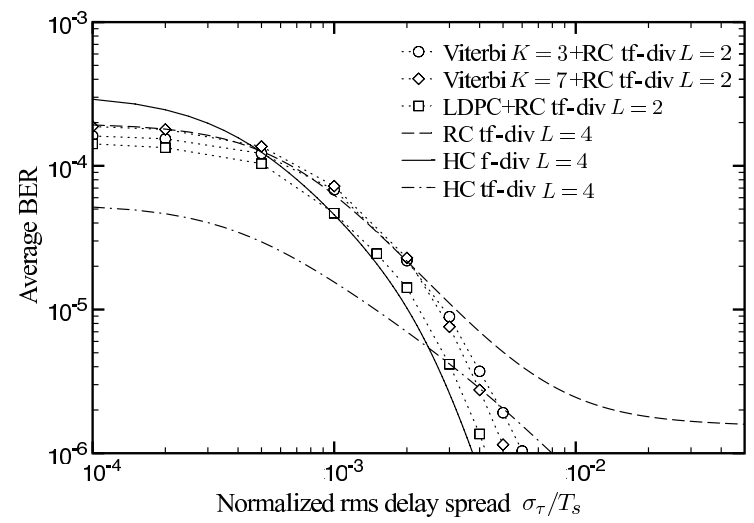

(b) $L=4, \Gamma=30 \mathrm{~dB}, f_{D} T_{s}=0.005$

Fig. 7 Comparison of average BER performance between repetition coding and error correcting coding considering variation of delay spread.

ing fluctuation, which is analytically verified by the performances without fading correlation calculated by (38) in Fig. 7(a). In such an environment as low fading correlation, the error correcting coding has an advantage over the repetition coding. On the other hand, in the case of $L=4$, the Hermite-symmetric repetition coding outperforms the others. It should be noted that the effect of error correcting coding varies depending on the coding/decoding technique, then the performance comparison in Figs. 7 and 8 is no more than an example. We would like to emphasize that the average BER performance in the repetition coded OFDM systems can be easily but precisely obtained by using the derived analytical equations, without Monte Carlo simulation.

\section{Conclusion}

In this paper, we theoretically analyzed the effect of the repetition coding in the OFDM systems by increasing the number of repetitions $L$ and acquiring both time and frequency diversity gains (two-dimensional diversity gain), and then derived closed-form equations of the average BER. Moreover, the effectiveness of repetition coding has been analytically verified depending on the wireless channel condition in a comparison with error correcting coding. We would like to search for the utilization of the repetition coding and

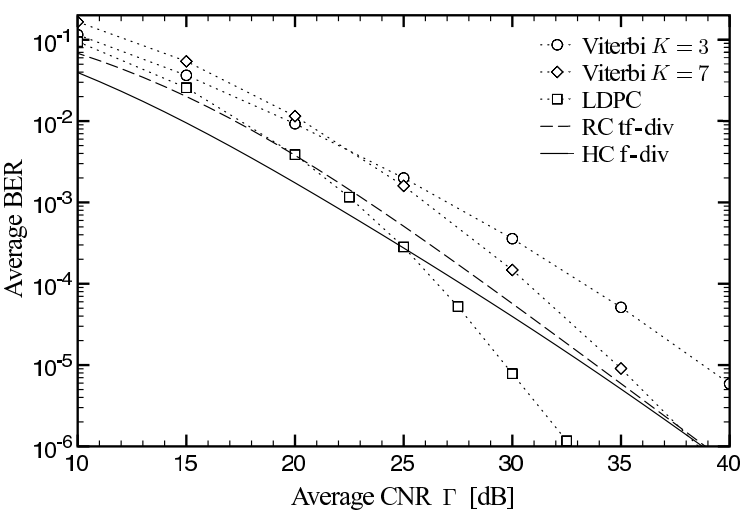

(a) $L=2, \sigma_{\tau} / T_{s}=0.001, f_{D} T_{s}=0.005$

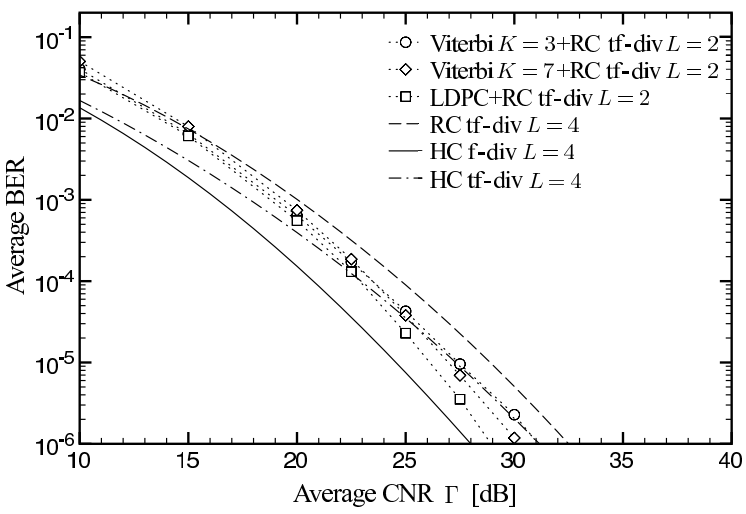

(b) $L=4, \sigma_{\tau} / T_{s}=0.001, f_{D} T_{s}=0.005$

Fig. 8 Comparison of average BER performance between repetition coding and error correcting coding considering variation of average CNR.

its analytical equation for various wireless communication systems. In addition, considering the computational complexity, we would like to search for further comparison with variable-rate (punctured) error correcting coding, hybrid automatic repeat request (HARQ), and so on.

\section{Acknowledgment}

This work was supported by JSPS KAKENHI Grant Number 24560448 .

\section{References}

[1] S.B. Weinstein and P.M. Ebert, "Data transmission by frequencydivision multiplexing using the discrete Fourier transform," IEEE Trans. Commun., vol.COM-19, no.5, pp.628-634, Oct. 1971.

[2] J.A.C. Bingham, "Multicarrier modulation for data transmission: An idea whose time has come," IEEE Commun. Mag., vol.28, no.5, pp.5-14, May 1990.

[3] S.M. Alamouti, "A simple transmit diversity technique for wireless communications," IEEE J. Sel. Areas Commun., vol.16, no.8, pp.1451-1458, Oct. 1998.

[4] D. Agrawal, V. Tarokh, A. Naguib, and N. Seshadri, "Space-time coded OFDM for high data-rate wireless communication over wideband channels," Proc. IEEE VTC'98, vol.3, pp.2232-2236, May 1998.

[5] K.F. Lee and D.B. Williams, "A space-frequency transmitter diversity technique for OFDM systems," Proc. IEEE GLOBECOM'00, 
vol.3 pp.1473-1477, Nov. 2000.

[6] Y.H. Kim, I. Song, H.G. Kim, T. Chang, and H.M. Kim, "Performance analysis of a coded OFDM system in time-varying multipath Rayleigh fading channels," IEEE Trans. Veh. Technol., vol.48, no.5, pp.1610-1615, Sept. 1999.

[7] J. Zheng and S.L. Miller, "Performance analysis of coded OFDM systems over frequency-selective fading channels," Proc. IEEE GLOBECOM2003, vol.3, pp.1623-1627, Dec. 2003.

[8] H. Futaki and T. Ohtsuki, "Performance of low-density paritycheck (LDPC) coded OFDM systems," Proc. IEEE ICC 2002, vol.3, pp.1696-1700, May 2002.

[9] S. Ye, R.S. Blum, and L.J. Cimini, Jr., "Adaptive modulation for variable-rate OFDM systems with imperfect channel information," Proc. IEEE VTC Spring 2002, vol.2, pp.767-771, May 2002.

[10] T. Usui, F. Ishizu, and K. Murakami, "A study on adaptive modulation technique in OFDM," IEICE Trans. Commun. (Japanese Edition), vol.J88-B, no.3, pp.634-642, March 2005.

[11] I. Barhumi, G. Leus, and M. Moonen, "Optimal training sequences for channel estimation in MIMO OFDM systems in mobile wireless channels," 2002 International Zurich Seminar on Broadband Commun., Access, Transmission, Networking, pp.44-1-44-6, Feb. 2002.

[12] Y. Jing, D. Wang, M. Chen, S. Cheng, and H. Wang, "Reduced complexity MIMO-OFDM channel estimation based on parametric channel model," Proc. IEEE VTC2004-Fall, vol.2, pp.1372-1376, Sept. 2004

[13] F. Sasamori, Y. Ishikawa, S. Handa, and S. Oshita, "A simple expression of BER performance in COFDM systems over fading channels," IEICE Trans. Fundamentals, vol.E92-A, no.1, pp.332-336 Jan. 2009.

[14] F. Sasamori, Z. Jia, S. Handa, and S. Oshita, "Performance analysis of repetition coded OFDM systems with diversity combining and higher-level modulation,” IEICE Trans. Commun., vol.E94-B, no.1, pp.194-202, Jan. 2011.

[15] F. Sasamori and S. Handa, "Performance analysis of Hermitesymmetric subcarrier coding for OFDM systems over fading channels," IEICE Trans. Fundamentals, vol.E95-A, no.12, pp.24612469, Dec. 2012.

[16] IEEE P802.16-REVd - Standard for Local and Metropolitan Area Networks - Part 16: Air Interface for Fixed Broadband Wireless Access Systems.

[17] J. Tubbax, B. Come, L.V. der Perre, S. Donnay, M. Engels, H.D. Man, and M. Moonen, "Compensation of IQ imbalance and phase noise in OFDM systems," IEEE Trans. Wireless Commun., vol.4, no.3, pp.872-877, May 2005.

[18] R. Corvaja and A.G. Armada, "Joint channel and phase noise compensation for OFDM in fast-fading multipath applicaitons," IEEE Trans. Veh. Technol., vol.58, no.2, pp.636-643, Feb. 2009.

[19] W.C. Jakes, Microwave Mobile Communications, John Wiley \& Sons, 1974

[20] Y. Karasawa, Radiowave Propagation Fundamentals for Digital Mobile Communications, Corona Publishing, Tokyo, 2003. (in Japanese)

[21] J.G. Proakis, Digital Communications, McGraw-Hill, 1983.

[22] W.C.Y. Lee, Mobile Communications Engineering, McGraw-Hill, 1982 .

[23] M. Abramowitz and I.A. Segun, Handbook of Mathematical Functions, Dover publications, 1965.

[24] P.A. Bello, "Characterization of randomly time-variant linear channels," IEEE Trans. Commun. Syst., vol.CS-11, no.4, pp.360-393, Dec. 1963.

[25] M. Patzold and A. Szczepanski, "Methods for modeling of specified and measured multipath power delay profiles," Proc. IEEE VTC2000-Spring, vol.3, pp.1828-1834, May 2000.

[26] BRAN WG3 PHY Subgroup, "Criteria for comparison," ETSI/ BRAN document no.30701F, 1998.

\section{Appendix A: Cardano's Solution for Cubic Equation and Its Rearrangement for BER Analy- sis}

A cubic equation

$$
x^{3}+a_{2} x^{2}+a_{1} x+a_{0}=0
$$

can be solved by using Cardano's solution as follows:

$$
\begin{aligned}
& x_{1}=\omega_{1} \sqrt[3]{-q+\sqrt{q^{2}+p^{3}}}+\omega_{1} \sqrt[3]{-q-\sqrt{q^{2}+p^{3}}}-\frac{a_{2}}{3}, \\
& x_{2}=\omega_{2} \sqrt[3]{-q+\sqrt{q^{2}+p^{3}}}+\omega_{3} \sqrt[3]{-q-\sqrt{q^{2}+p^{3}}}-\frac{a_{2}}{3}, \\
& x_{3}=\omega_{3} \sqrt[3]{-q+\sqrt{q^{2}+p^{3}}}+\omega_{2} \sqrt[3]{-q-\sqrt{q^{2}+p^{3}}}-\frac{a_{2}}{3},
\end{aligned}
$$

where

$$
\begin{aligned}
& \omega_{1}=1, \\
& \omega_{2}=\frac{-1+j \sqrt{3}}{2}=\exp \left(j \frac{2 \pi}{3}\right), \\
& \omega_{3}=\frac{-1-j \sqrt{3}}{2}=\exp \left(-j \frac{2 \pi}{3}\right), \\
& p=\frac{a_{1}}{3}-\frac{a_{2}^{2}}{9}, \\
& q=\frac{a_{0}}{2}+\frac{a_{2}^{3}}{27}-\frac{a_{2} a_{1}}{6} .
\end{aligned}
$$

A correlation matrix in Sect. 3.2 is classified into Hermite matrix whose eigenvalues ought to be a real number. But $q^{2}+p^{3}$ in (A.2) always becomes a negative value, then the terms in (A.2) should be rewritten as

$$
\begin{aligned}
& \sqrt[3]{-q+\sqrt{q^{2}+p^{3}}} \\
& =\sqrt[3]{-q+j \sqrt{-q^{2}-p^{3}}} \\
& =\sqrt{-p} \exp \left\{j \frac{1}{3} \tan ^{-1}\left(\frac{\sqrt{-q^{2}-p^{3}}}{-q}\right)\right\}, \\
& \sqrt[3]{-q-\sqrt{q^{2}+p^{3}}} \\
& =\sqrt{-p} \exp \left\{-j \frac{1}{3} \tan ^{-1}\left(\frac{\sqrt{-q^{2}-p^{3}}}{-q}\right)\right\} .
\end{aligned}
$$

By using Euler's formula, the solutions (A-2) for the cubic Eq. (A. 1) can be rewritten as

$$
\begin{aligned}
& x_{1}=2 \sqrt{-p} \cos \left\{\frac{1}{3} \tan ^{-1}\left(\frac{\sqrt{-q^{2}-p^{3}}}{-q}\right)\right\}-\frac{a_{2}}{3}, \\
& x_{2}=2 \sqrt{-p} \cos \left\{\frac{1}{3} \tan ^{-1}\left(\frac{\sqrt{-q^{2}-p^{3}}}{-q}\right)+\frac{2 \pi}{3}\right\}-\frac{a_{2}}{3},
\end{aligned}
$$




$$
x_{3}=2 \sqrt{-p} \cos \left\{\frac{1}{3} \tan ^{-1}\left(\frac{\sqrt{-q^{2}-p^{3}}}{-q}\right)-\frac{2 \pi}{3}\right\}-\frac{a_{2}}{3} \text {. }
$$

\section{Appendix B: Euler's Solution for Quartic Equation}

A quartic equation

$$
y^{4}+b_{2} y^{2}+b_{1} y+b_{0}=0
$$

can be solved by using Euler's solution. First, a resolvent cubic equation

$$
x^{3}+\frac{b_{2}}{2} x^{2}+\left(\frac{b_{2}^{2}}{16}-\frac{b_{0}}{4}\right) x-\frac{b_{1}^{2}}{64}=0,
$$

which is derived from (A. 6), is solved by using Cardano's solution in Appendix A. Next, the quartic Eq. (A. 6) can be solved by using the solutions $x_{i}(i=1,2,3)$. If $b_{1} \leq 0$, the solutions for the quartic equation $(\mathrm{A} \cdot 6)$ become

$$
\begin{aligned}
& y_{1}=\sqrt{x_{1}}+\sqrt{x_{2}}+\sqrt{x_{3}}, \\
& y_{2}=\sqrt{x_{1}}-\sqrt{x_{2}}-\sqrt{x_{3}}, \\
& y_{3}=-\sqrt{x_{1}}+\sqrt{x_{2}}-\sqrt{x_{3}}, \\
& y_{4}=-\sqrt{x_{1}}-\sqrt{x_{2}}+\sqrt{x_{3}} .
\end{aligned}
$$

On the other hand, if $b_{1}>0$, the solutions become

$$
\begin{aligned}
& y_{1}=-\sqrt{x_{1}}-\sqrt{x_{2}}-\sqrt{x_{3}}, \\
& y_{2}=-\sqrt{x_{1}}+\sqrt{x_{2}}+\sqrt{x_{3}}, \\
& y_{3}=+\sqrt{x_{1}}-\sqrt{x_{2}}+\sqrt{x_{3}}, \\
& y_{4}=+\sqrt{x_{1}}+\sqrt{x_{2}}-\sqrt{x_{3}} .
\end{aligned}
$$

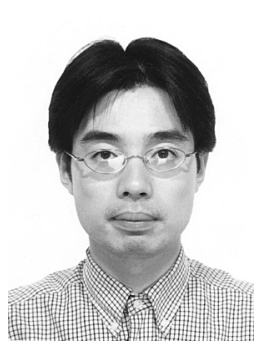

Fumihito Sasamori received the B.E., M.E. and Dr.Eng. Degrees from Waseda University, Tokyo, Japan in 1994, 1996 and 2000, respectively. Since 2000 he has been with the Department of Electrical and Electronic Engineering, Shinshu University, first as a Research Associate and since 2006 as an Associate Professor. His current research interests include digital mobile communication systems. He received the IEICE Young Engineer Award in 2000. He is a member of IEEE.

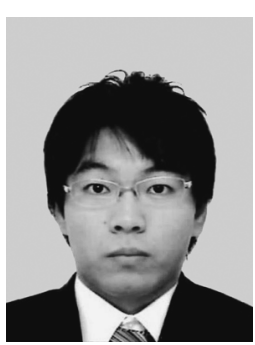

Satoru Asada received the B.E. and M.E. degrees from Shinshu University, Nagano, Japan in 2010 and 2012 respectively. He has been with Central Japan Railway Company, Japan.

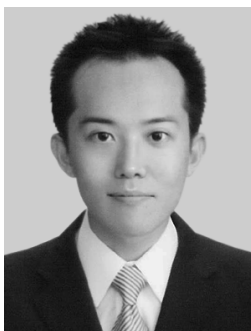

Osamu Takyu received the B.E. in Electrical Engineering from Tokyo University of Science, Chiba, Japan in 2002, and the M.E. and Ph.D. degrees in Open and Environmental systems from Keio University, Yokohama, Japan in 2003 and 2006, respectively. From 2003 to 2007, he was a Research Associate in the Department of Information and Computer Science, Keio University. From 2004 to 2005, he was visiting scholar in the School of Electrical and Information Engineering, University of Sydney.

From 2007 to 2010, he was an Assistant Professor in the Department of Electrical Engineering, Tokyo University of Science. Since 2011 he has been with the Department of Electrical and Electronic Engineering, Shinshu University, first as a Research Associate and since 2013 as an Associate Professor. His current research interests are in wireless communication systems and distributed wireless communication technology. He is a member of IEEE.

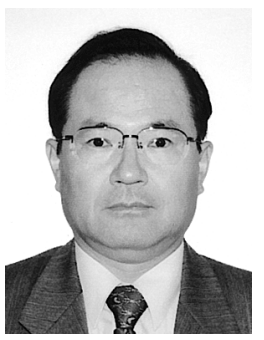

Shiro Handa received the B.E. and M.E. degrees from Shinshu University in 1978 and 1980 respectively, and the Dr.Eng. degree from Kobe University in 1988. From 1982 to 1988, he was a Research Associate at Kobe University. From 1988 to 1994, he was with Nagano National College of Technology. He has been with the Department of Electrical and Electronic Engineering, Shinshu University, since 1994 as an Associate Professor and since 2005 as a Professor In 1996, he was at the University of California, Davis, as a visiting researcher. His research interests include satellite and mobile communication systems, modulation and coding, and visible light communications. He is a member of IEEE. 